\title{
Construct Validity of the Short Form-36 Health Survey and Its Relationship with BMI in Obese Outpatients
}

\author{
Francesco Corica, * Andrea Corsonello, $* \dagger$ Giovanni Apolone, $\neq$ Maria Lucchetti, $\dagger$ Nazario Melchionda, $\S$ \\ Giulio Marchesini, $\S$ and the QUOVADIS Study Group
}

\begin{abstract}
CORICA, FRANCESCO, ANDREA CORSONELLO, GIOVANNI APOLONE, MARIA LUCCHETTI, NAZARIO MELCHIONDA, GIULIO MARCHESINI, AND THE QUOVADIS STUDY GROUP. Construct validity of the Short Form-36 Health Survey and its relationship with BMI in obese outpatients. Obesity. 2006; 14:1429-1437.
\end{abstract}

Objective: To investigate the construct validity of the Short Form-36 (SF-36) Health Survey questionnaire in obese patients.

Research Methods and Procedures: Our series consisted of 1735 obese patients (age, $44.7 \pm 11.0$ years; 1346 women) consecutively enrolled in the QUOVADIS study, an observational multicenter study of obese treatment-seeking outpatients. The construct validity of the SF-36 was assessed by main component analysis. Age-, gender-, and educationadjusted general linear models were used to investigate the relationship between BMI and SF-36 domains or factors identified by main component analysis.

Results: BMI was significantly associated with poor healthrelated quality of life in all eight SF-36 domains, and the strongest association was observed with physical activity. Main components analysis generated a six-factor solution

Received for review December 5, 2005

Accepted in final form June 5, 2006.

The costs of publication of this article were defrayed, in part, by the payment of page charges. This article must, therefore, be hereby marked "advertisement" in accordance with 18 U.S.C. Section 1734 solely to indicate this fact.

*Department of Internal Medicine, University of Messina, Messina, Italy; †Italian National Research Center on Aging, Ancona, Cosenza, Italy; $\$$ Clinical Research Laboratory, Institute of Pharmacological Research Mario Negri, Milan, Italy; and q[Metabolic Unit, Alma Mater Studiorum University, Bologna, Italy.

Address correspondence to Andrea Corsonello, Viale della Resistenza, Pal. Alfa Scala H, I-87036 Rende (CS), Italy.

E-mail: andrea_corsonello@tin.it

Copyright () 2006 NAASO explaining $59 \%$ of the observed variance. BMI was strongly associated with factors based on the loading of items regarding the physical activity domain and factors based on role-physical and role-emotional items or general health and bodily pain items. In contrast, mental health-, vitality-, and social functioning-based factors were not related to BMI.

Discussion: In obese treatment-seeking outpatients, the clustering of SF-36 items in main components is not significantly different from the domain-based approach generally used, thus confirming the robustness of such a generic questionnaire in this specific condition. However, the peculiar clustering of some SF-36 items and their relationship with BMI suggest that the health-related quality of life profile of subjects belonging to that population may be better described with alternative aggregations of the SF-36 items or with disease-tailored questionnaires.

Key words: construct validity, Short Form-36 Health Survey, health-related quality of life, observational study, main component analysis

\section{Introduction}

Obesity is a complex, multifactorial disease whose etiology involves genetic, metabolic, social, behavioral, and cultural factors (1). Several studies have shown that obesity has a significant impact on morbidity and mortality, as well as psychosocial well-being and quality of life, thus yielding the measurement of health-related quality of life (HRQL), ${ }^{1}$ an emerging topic in obesity studies (2).

Available evidence suggests that the Short Form-36 (SF36) Health Survey questionnaire (3) is a valid generic measure for rating HRQL in several research fields on the basis of its good construct validity, high internal consistency, and

\footnotetext{
${ }^{1}$ Nonstandard abbreviations: HRQL, health-related quality of life; SF-36, Short Form-36; BP, bodily pain; GH, general health; VT, vitality; SF, social functioning; $\mathrm{MH}$, mental health.
} 
high test-retest reliability (4-6). Furthermore, the SF-36 Health Survey is easy to administer and to compile for respondents, has extensive psychometric validation, and is responsive to treatment in several medical conditions, including obesity (7). A lot of studies have been carried out in obese patients using the SF-36 Health Survey questionnaire, but results are not unequivocal. When tested by the SF-36 Health Survey, HRQL was shown to be impaired with the severity of obesity among individuals seeking non-surgical weight loss treatment, with the most obese individuals having the poorest quality of life (8) and obese persons reporting pain showing the greatest impairments (9). Fontaine et al. (8) reported that obese participants in an outpatient weight loss program scored significantly lower than healthy subjects on all eight domains of the SF-36 Health Survey, thus suggesting an unspecific interaction between obesity and HRQL. Yancy et al. (10), however, showed that increasing BMI affected mainly physical activity and bodily pain (BP) domains, and Doll et al. (11) showed that obesity had a particularly negative impact on physical well-being. In an Italian series, reduced scores on the SF-36 Health Survey in obesity were also related to psychological distress (12) and binge eating (13).

Because the SF-36 Health Survey has been developed and validated in a general population $(3,5,14,15)$, we can expect the existence of an obesity-specific hierarchy of cumulatively considered SF-36 Health Survey items that might help to clarify the real impact of obesity on HRQL. The QUOVADIS program is an observational multicenter study aimed specifically at measuring the burden of obesity and its complications on HRQL and psychological distress (14). It represents a valuable opportunity to investigate this issue in a large population sample of obese subjects seeking medical treatment at medical centers. We investigated the internal structure of the SF-36 Health Survey scale to ascertain the existence of distinctive HRQL multiple dimensions, i.e., different combinations of items, in this population, and to investigate their relationship with BMI. If distinctive multiple dimensions were present inside the SF-36 Health Survey in this specific setting, then the effects of obesity on HRQL would be different from those observed on the basis of the classical SF-36 Health Survey 8 domain scores.

\section{Research Methods and Procedures}

\section{Patients}

The present report uses data from the QUOVADIS study, which has been described in detail elsewhere (16). Briefly, during the years 1999 to 2000, 25 Italian centers with specific interest in clinical research consecutively enrolled treatment-seeking obese patients $\left(\mathrm{BMI}>30 \mathrm{~kg} / \mathrm{m}^{2}\right)$ in the study. The enrollment period was preceded by two meetings of the steering committee and by a general investigators' meeting to decide selection criteria and to agree on data collection and management of patients. All obese subjects seeking treatment were eligible for the study, provided that they were not on active treatment for obesity at the time of enrollment, were in the age range between 20 and 65, agreed to fill out a package of self-administered questionnaires, and signed an informed consent to participate.

History taking and clinical examination were performed by means of a predefined checklist agreed upon by all participants. The Case Report Form included information on civil and educational status, personal and family history of metabolic and cardiovascular diseases, and previous and current pharmacological treatment. To expedite handling of data, the study was totally web-based through an extranet system provided by CINECA (Casalecchio di Reno, Italy), using the Advanced Multicenter Research methodology developed by CINECA as a result of an extensive cooperation among clinicians, statisticians, and informatics. The management of all the data was performed by standard web browsers, and the quality level was guaranteed by upfront quality controls (on the client side) and consistency checks (on the server side). Overall, 1944 obese patients were enrolled in the study period, but complete data for the present study were available in only 1735 cases. On enrollment, all subjects signed an informed consent to take part in the study, which was approved by the Ethical Committee of the coordinating center (Bologna) and by Institutional Review Boards of individual local centers.

\section{Definition of the Outcome}

The main outcome of the study was HRQL, measured by the SF-36 Health Survey questionnaire $(3,5)$. SF-36 is a 36-item questionnaire measuring subjective health status. Response items are usually arranged in eight domains reflecting physical and mental HRQL: physical functioning, role limitations (because of physical problems), BP, general health $(\mathrm{GH})$, vitality (VT), social functioning (SF), role limitations (because of emotional problems), and mental health $(\mathrm{MH})$.

\section{Measurements}

Body weight was measured in light clothing and without shoes to the nearest $0.5 \mathrm{~kg}$. Height was measured to the nearest $0.5 \mathrm{~cm}$. BMI was calculated as weight (in kilograms) divided by squared height (in meters). Other variables specifically considered in the analysis were age, gender, and education.

\section{Analytic Approach}

First, age-, gender-, and education-adjusted general linear model analysis was performed to obtain a deconfounded 
estimate of the relationship between BMI and the classical eight domains of the SF-36 Health Survey.

The construct validity of the SF-36 Health Survey scale was then assessed by the main component analysis (17). As a first step, we tested the appropriateness of the main component analysis by the Kaiser-Meyer-Olkin Measure of Sampling Adequacy and the Bartlett's test of sphericity (18). Commonalities (the squared multiple correlation coefficients between a variable and all other variables) were assumed to reflect the strength of the linear association among the variables.

The basic assumption of factor analysis is that complex phenomena, such as the SF-36-based profile of quality of life, can be explained by underlying dimensions or factors. Factors corresponding to a set of closely interrelated SF-36 Health Survey items were identified by the main component analysis (factor extraction). The component matrix was obtained, and then a rotated component matrix was derived through an oblique rotation. The component matrix represents the crude relationship between the factors and the individual SF-36 Health Survey items, but it fails to disclose an easily interpretable pattern of correlation because many items have moderate size correlations with most factors. In contrast, the oblique rotation simplifies the relationship between individual items and factors by minimizing the number of SF-36 Health Survey items having loadings on a factor. The rotation phase makes the initial matrix easier to interpret, grouping SF-36 Health Survey items to identify factors corresponding to well-defined dimensions of HRQL. The strength of the relationship between factors and items was assumed to be directly proportional to the magnitude of the coefficient relating individual items to the extracted factor. Furthermore, the oblique rotation provides a measure of correlations among factors.

We identified factors to be retained in the final model on a graphical plot of explained variance by corresponding factor; retained factors can be recognized as those preceding the boundary between factors explaining large and small proportions of variance (17). A scale that explains most of the observed variance by a few factors has a solid internal structure; i.e., chance variations are unlikely to confound the interpretation of the differences observed both within and between groups.

The crude prevalence of impaired HRQL in factors identified by main component analysis (i.e., the prevalence of the poorest score for each item having loading with a given factor) in relation to different BMI levels was evaluated by cross-tabulations and Pearson's $\chi^{2}$. Finally, the association between BMI and factors identified with main component analysis was investigated by age-, gender-, and educationadjusted general linear model analysis. All analyses were performed using SPSS version 10.0 (SPSS Inc., Chicago, IL).
Table 1. Demographic and anthropometric characteristics of the population studied

\begin{tabular}{lc}
\hline & $N=\mathbf{1 7 3 5}$ \\
\hline Age (years) & $44.7 \pm 11.0$ \\
Gender (females) & $1346(77.6)$ \\
Education & \\
$\quad$ Illiterate & $31(1.8)$ \\
Primary school & $283(16.3)$ \\
Secondary school & $568(32.7)$ \\
Commercial or vocational school & $709(40.9)$ \\
Academic degree & $144(8.3)$ \\
BMI (kg/m $\left.{ }^{2}\right)$ & \\
30 to 34.9 & $678(39.1)$ \\
35 to 39.9 & $497(28.6)$ \\
40 to 44.9 & $333(19.2)$ \\
$\geq 45$ & $227(13.1)$ \\
\hline
\end{tabular}

Data are mean \pm standard deviation or number of cases with percentage in parentheses.

\section{Results}

General characteristics of the population studied are reported in Table 1. The majority of patients were women, and $\sim 32 \%$ of patients had severe obesity with a BMI $\geq 40$ $\mathrm{kg} / \mathrm{m}^{2}$.

BMI was significantly associated with poor HRQL in all SF-36 Health Survey domains; the strongest association was observed with physical activity, whereas the weakest one was found with role-emotional (Table 2).

A main component analysis was feasible in our population. The high Kaiser-Meyer-Olkin measure of sampling adequacy $(>0.9)$ confirmed the hypothesis that the correlations between pairs of variables could be explained by the other variables. The highly significant Bartlett's test of sphericity $(p<0.001)$ denied that the item correlation matrix was an identity matrix. Main components and explained variance are reported in Table 3, and a six-factor solution explaining $59 \%$ of the observed variance was generated.

Table 4 shows the rotated component matrix and total variance explained by Factors 1 to 6 in the whole group. Factors 2 and 6 were based on the loading of items regarding the SF-36 Health Survey physical activity domain. In particular, items exploring the performance in vigorous activities and complex movements had a separate loading on Factor 6. Items exploring role-physical and role-emotional had loading on Factor 3, while those exploring GH and BP had loading on Factor 4. Factor 1 had loading with negative items of MH (Have you been very nervous? Have you felt so down in the dumps that nothing could cheer you 
Table 2. Multivariable general linear model* for the relationship between SF-36 Health Survey scores and BMI

\begin{tabular}{|c|c|c|c|}
\hline \multirow[b]{2}{*}{ SF-36 scores } & \multicolumn{3}{|c|}{ BMI } \\
\hline & $F$ & $\begin{array}{l}\text { Adjusted } \\
\qquad R^{2}\end{array}$ & $p$ \\
\hline Physical activity & 59.210 & 0.12 & 0.001 \\
\hline Physical role & 14.912 & 0.03 & 0.001 \\
\hline Pain & 12.156 & 0.02 & 0.001 \\
\hline GH & 18.762 & 0.04 & 0.001 \\
\hline VT & 5.819 & 0.01 & 0.001 \\
\hline Social activity & 4.879 & 0.01 & 0.001 \\
\hline Emotional role & 3.068 & 0.01 & 0.016 \\
\hline MH & 4.250 & 0.01 & 0.002 \\
\hline
\end{tabular}

SF-36, Short Form-36; GH, general health; VT, vitality; MH, mental health.

* After adjusting for age, gender, and education.

up? Have you felt downhearted and depressed?) and VT domains (Did you feel worn out? Did you feel tired?). Factor 5 had loading with positive items exploring SF, MH (Have you felt calm and peaceful? Have you been happy?), and VT (Did you feel full of life? Did you have a lot of energy?).

The crude prevalence of impaired HRQL in Factors 2 and 6 was strongly associated with increasing BMI, and a similar linear trend was observed when considering Factors 3 and 4 (Figure 1). Adjusted analysis confirmed that BMI was strongly associated with Factor 6 ( $F$ value, 35.482) and
Factor 2 ( $F$ value, 20.407) and with Factors 3 and 4. On the other hand, Factors 1 and 5, i.e., items exploring MH, VT, and SF, did not show any significant correlation with BMI (Table 5).

\section{Discussion}

Generic scales for HRQL measurement are designed to be a reference instrument, allowing a disease to be situated in relation to all other diseases, and can be used to compare obesity and its consequences with other chronic diseases. In contrast, disease-specific scales are conceptualized and operationalized to emphasize elements that discriminate a given population from a control or general population. In view of its characteristics, an obesity-specific instrument for measuring HRQL can be used only to assess differences in HRQL within the obese population $(19,20)$. Results from the present study are somewhat in agreement with the hypothesis that a complementary approach based on both generic and specific instruments would better define the impact obesity has on HRQL and seems to be recommendable (20).

The pattern of segregation of SF-36 Health Survey items deserves consideration. The items exploring physical activity were segregated in two main components, based on vigorous activities and complex movements (Factor 6) and all other physical activities (Factor 2), respectively. This particular clustering of physical activity items may correspond to different degrees of obesity-related physical impairment, with less impaired patients having limitations only in highly demanding physical activities and, at the opposite extreme, more impaired patients having limitations in mild-to-moderate physical functions having load on Factor 2. These findings might have important implications for the management of obese patients because they identify

Table 3. Main components identified by factor analysis and percentage of variance explained in patients studied

\begin{tabular}{|c|c|c|c|c|c|c|c|}
\hline \multirow[b]{2}{*}{ Component } & \multicolumn{3}{|c|}{ Initial Eigenvalues } & \multicolumn{3}{|c|}{ Extraction sums of squared loadings } & \multirow{2}{*}{$\begin{array}{c}\text { Rotated sums } \\
\text { of squared } \\
\text { loadings: } \\
\text { total }\end{array}$} \\
\hline & Total & $\begin{array}{c}\text { Variance } \\
(\%)\end{array}$ & $\begin{array}{c}\text { Cumulative } \\
(\%)\end{array}$ & Total & $\begin{array}{c}\text { Variance } \\
(\%)\end{array}$ & $\begin{array}{c}\text { Cumulative } \\
(\%)\end{array}$ & \\
\hline 1 & 11.253 & 32.151 & 32.151 & 11.253 & 32.151 & 32.151 & 6.187 \\
\hline 2 & 3.530 & 10.087 & 42.238 & 3.530 & 10.087 & 42.238 & 6.449 \\
\hline 3 & 1.898 & 5.422 & 47.660 & 1.898 & 5.422 & 47.660 & 6.947 \\
\hline 4 & 1.661 & 4.746 & 52.406 & 1.661 & 4.746 & 52.406 & 5.215 \\
\hline 5 & 1.286 & 3.675 & 56.081 & 1.286 & 3.675 & 56.081 & 5.870 \\
\hline 6 & 1.084 & 3.097 & 59.178 & 1.084 & 3.097 & 59.178 & 2.445 \\
\hline
\end{tabular}

Only factors preceding the boundary between factors explaining large and small fractions of the global variance in Short Form-36 Health Survey are reported. Extraction method: main component analysis. 
Table 4. Rotated structure matrix in the population studied

\begin{tabular}{lcccccr}
\hline \multicolumn{1}{c}{ Items } & \multicolumn{5}{c}{ Factor } \\
\cline { 2 - 7 } & $\mathbf{1}$ & $\mathbf{2}$ & $\mathbf{3}$ & $\mathbf{4}$ & $\mathbf{5}$ & $\mathbf{6}$ \\
\hline 1. In general, would you say your health is: & -0.319 & -0.356 & -0.330 & $\mathbf{0 . 7 0 8}$ & -0.345 & -0.281 \\
3. The following questions are about activities & & & & & & \\
you might do during a typical day. Does your & & & & & & \\
health now limit you in these activities? If so, & & & & & & \\
how much? & & & & & & \\
a. Vigorous activities, such as running, etc. & 0.108 & 0.287 & 0.276 & -0.257 & 0.389 & $\mathbf{0 . 6 1 0}$ \\
b. Moderate activities, such as moving a table, & & & & & & \\
$\quad$ etc. & 0.235 & $\mathbf{0 . 6 7 3}$ & 0.383 & -0.356 & 0.225 & 0.381 \\
c. Lifting or carrying groceries & 0.302 & $\mathbf{0 . 6 3 3}$ & 0.353 & -0.357 & 0.138 & 0.454 \\
d. Climbing several flights of stairs & 0.296 & $\mathbf{0 . 6 3 2}$ & 0.309 & -0.238 & 0.191 & 0.559 \\
e. Climbing one flight of stairs & 0.286 & $\mathbf{0 . 7 5 0}$ & 0.282 & -0.251 & 0.007 & 0.324 \\
f. Bending, kneeling, or stooping & 0.228 & 0.556 & 0.301 & -0.273 & 0.189 & $\mathbf{0 . 5 6 9}$ \\
g. Walking more than a mile & 0.250 & $\mathbf{0 . 7 5 5}$ & 0.296 & -0.302 & 0.200 & 0.338 \\
h. Walking several hundred yards & 0.202 & $\mathbf{0 . 8 7 2}$ & 0.247 & -0.271 & 0.133 & 0.009 \\
i. Walking 100 yards & 0.178 & $\mathbf{0 . 8 2 8}$ & 0.225 & -0.258 & 0.009 & -0.004 \\
1. Bathing or dressing yourself & 0.146 & $\mathbf{0 . 6 9 6}$ & 0.286 & -0.255 & 0.106 & -0.006
\end{tabular}

4. During the past 4 weeks, how much of the time have you had any of the following problems with your work or other regular daily activities as a result of your physical health?

a. Cut down on the amount of time you spent on work or other activities

b. Accomplished less than you would like

c. Were limited in the kind of work or other activities

$\begin{array}{llllll}0.125 & 0.351 & \mathbf{0 . 7 3 9} & -0.327 & 0.161 & 0.008 \\ 0.355 & 0.251 & \mathbf{0 . 7 9 5} & -0.290 & 0.295 & 0.241 \\ & & & & & \\ 0.220 & 0.341 & \mathbf{0 . 7 7 9} & -0.380 & 0.153 & 0.235 \\ & & & & & \\ 0.381 & 0.258 & \mathbf{0 . 7 2 5} & -0.343 & 0.242 & 0.300\end{array}$

5. During the past 4 weeks, how much of the time have you had any of the following problems with your work or other regular daily activities as a result of any emotional problems (such as feeling depressed or anxious)?

a. Cut down on the amount of time you spent on work or other activities

$\begin{array}{llllll}0.323 & 0.297 & \mathbf{0 . 7 1 1} & -0.207 & 0.375 & -0.158 \\ 0.460 & 0.169 & \mathbf{0 . 7 0 5} & -0.180 & 0.408 & -0.006 \\ & & & & & \\ 0.496 & 0.140 & \mathbf{0 . 5 6 4} & -0.137 & 0.438 & -0.144\end{array}$

6. During the past 4 weeks, to what extent has your physical health or emotional problems interfered with your normal social activities with family, friends, neighbors, or groups?

7. How much BP have you had during the past 4 weeks?

$\begin{array}{llllll}-0.572 & -0.266 & -0.500 & 0.317 & \mathbf{- 0 . 5 9 5} & 0.007 \\ -0.452 & -0.295 & -0.453 & \mathbf{0 . 6 1 4} & -0.189 & -0.346\end{array}$


Table 4. Rotated structure matrix in the population studied (Continued)

\begin{tabular}{|c|c|c|c|c|c|c|}
\hline \multirow[b]{2}{*}{ Items } & \multicolumn{6}{|c|}{ Factor } \\
\hline & 1 & 2 & 3 & 4 & 5 & 6 \\
\hline $\begin{array}{l}\text { 8. During the past } 4 \text { weeks, how much did pain } \\
\text { interfere with your normal work (including } \\
\text { both work outside the home and housework)? }\end{array}$ & -0.484 & -0.390 & -0.581 & 0.642 & -0.230 & -0.290 \\
\hline $\begin{array}{l}\text { 9. These questions are about how you feel and } \\
\text { how things have been with you during the past } \\
4 \text { weeks. For each question, please give the } \\
\text { one answer that comes closest to the way you } \\
\text { have been feeling. How much of the time } \\
\text { during the past } 4 \text { weeks: }\end{array}$ & & & & & & \\
\hline a. Did you feel full of life? & -0.412 & -0.140 & -0.342 & 0.306 & -0.788 & -0.008 \\
\hline b. Have you been very nervous? & 0.710 & 0.200 & 0.294 & -0.212 & 0.444 & -0.144 \\
\hline $\begin{array}{l}\text { c. Have you felt so down in the dumps that } \\
\text { nothing could cheer you up? }\end{array}$ & 0.701 & 0.330 & 0.385 & -0.290 & 0.537 & -0.167 \\
\hline d. Have you felt calm and peaceful? & -0.522 & -0.127 & -0.306 & 0.299 & -0.767 & -0.003 \\
\hline e. Did you have a lot of energy? & -0.416 & -0.193 & -0.386 & 0.337 & -0.729 & -0.257 \\
\hline f. Have you felt downhearted and depressed? & 0.718 & 0.291 & 0.375 & -0.293 & 0.601 & -0.010 \\
\hline g. Did you feel worn out? & 0.787 & 0.282 & 0.347 & -0.272 & 0.295 & 0.122 \\
\hline h. Have you been happy? & -0.296 & -0.119 & -0.261 & 0.239 & -0.832 & -0.002 \\
\hline i. Did you feel tired? & 0.733 & 0.259 & 0.367 & -0.282 & 0.350 & 0.249 \\
\hline
\end{tabular}

10. During the past 4 weeks, how much of the time has your physical health or emotional problems interfered with your social activities (like visiting friends, relatives, etc.)?

$\begin{array}{llllll}0.549 & 0.306 & 0.433 & -0.310 & \mathbf{0 . 6 0 0} & -0.004\end{array}$

11. How TRUE or FALSE is each of the following statements for you?

a. I seem to get sick a little easier than other people

b. I am as healthy as anybody I know

c. I expect my health to get worse

d. My health is excellent

$\begin{array}{rrrrrr}0.316 & 0.274 & 0.299 & \mathbf{- 0 . 6 8 9} & 0.244 & -0.006 \\ -0.004 & -0.160 & -0.165 & \mathbf{0 . 6 5 3} & -0.142 & 0.002 \\ 0.173 & 0.229 & 0.185 & \mathbf{- 0 . 4 1 1} & 0.376 & 0.160 \\ -0.289 & -0.291 & -0.350 & \mathbf{0 . 7 6 0} & -0.410 & -0.264\end{array}$

Extraction method: main component analysis. Rotation method: Oblimin with Kaiser normalization. The greatest loading is reported in boldface to show the relationship with the main components.

Question 2 of the Short Form-36 was not considered in the analysis because it is a summary item and is not included in the eight domain scores.

clusters of items/functions that are lost or retained together. Theoretically, selected patients might regain a cluster of functions by a dedicated intervention. Thus, specific physical activity programs may be tailored to regain or maintain highly demanding physical functions or to approach wider defects of mild and moderate physical activities (21). Furthermore, identifying patients with a greater impairment in mild and moderate physical activities could have some prognostic relevance, considering the importance of a reg- ular physical exercise in the treatment of obesity and in weight loss maintenance (22).

Role limitations because of physical or emotional problems were aggregated in a single main component (Factor 3 ), as were items exploring GH and BP (Factor 4). Although it has been suggested that comorbidity may be a confounding variable in the association between BMI and emotional well-being (11), in a recent study, patients with obesity had significantly lower physical and emotional performance 


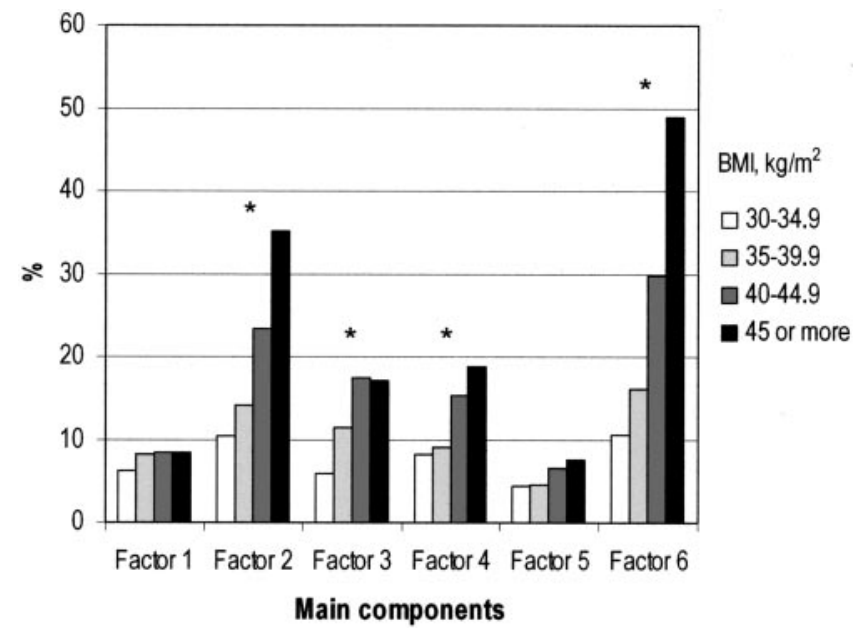

Figure 1: Crude prevalence of impaired HRQL in main components in relation to BMI. (Factor 1, items 9, b, c, f, g, and i; Factor 2, items 3, b to e and g to 1; Factor 3, items 4, a to d and 5, a to c; Factor 4, items 1, 7, 8, and 11, a to d; Factor 5, items 6, 9a, d, $\mathrm{e}, \mathrm{h}$, and 10; Factor 6, items 3 , a and f. $* p$ for linear trend $<0.001$.

than those who were normal weight, and such lower scores were seen even for persons without chronic comorbidities (23). These and our findings suggest that it may be difficult to disentangle physical and emotional effects of increasing BMI in a population of obese treatment-seeking patients when using a generic instrument for measuring HRQL. Furthermore, the greater prevalence of anxiety and depressive symptoms is a well-known feature of obese patients (24), and these problems may contribute to the parallel increase of the obesity-related physical and emotional role limitations. More simply, patients were not able to distinguish the actual difference between the different items exploring physical and emotional concepts in the context of a quite long survey they were asked to complete.

Table 5 Multivariable general linear model* for the relationship between main components identified by factor analysis and BMI

\begin{tabular}{lrcc}
\hline $\begin{array}{c}\text { SF-36 } \\
\text { scores }\end{array}$ & \multicolumn{3}{c}{$\begin{array}{c}\text { BMI } \\
\text { Adjusted } \boldsymbol{R}^{\mathbf{2}}\end{array}$} \\
\hline Factor 1 & 0.695 & 0.003 & $\boldsymbol{p}$ \\
Factor 2 & 20.407 & 0.08 & 0.595 \\
Factor 3 & 8.381 & 0.01 & 0.001 \\
Factor 4 & 5.977 & 0.01 & 0.001 \\
Factor 5 & 0.535 & 0.002 & 0.710 \\
Factor 6 & 35.482 & 0.10 & 0.001
\end{tabular}

SF-36, Short Form-36.

* After adjusting for age, gender, and education.
The aggregation between pain and GH perception in a single main component is less unexpected for several reasons; first of all, both scales pertain to the domain of physical health; then, BP is an important correlate of the subjective perception of health status (25); and finally, body weight is associated with disability, depression, and poor physical function in chronic pain patients (26), and pain is qualified as a strong covariate of obesity with a significant burden on functional health status and subjective well-being (27).

Finally, the VT, SF, and MH domains had loading on Factors 1 and 5. These results suggest that items regarding these three SF-36 Health Survey domains may have a reduced discriminatory capacity in this population of obese outpatients due to the generic approach of the scale referring to a universal and normative concept of the good mental and social functioning (20). Taking into account real situations experienced by the obese patient, as can be obtained when using a specific instrument for HRQL measurement, could herald different results and may contribute to a better definition of the relationship between increasing BMI and psychological-social well-being.

The relationship between the severity of obesity and HRQL was clearly different when considering SF-36 Health Survey domains or the results of the main component analysis, with all domains significantly affected in the first case and only main components regarding physical aspects, BP, $\mathrm{GH}$, and role-emotional significantly related to BMI in the second one. This finding suggests a plausible explanation for the apparent discrepancy among results obtained in previous studies $(8,10,11,23)$; at least in a population of obese treatment-seeking patients, the relationship between increasing BMI and HRQL could be affected by the particular internal structure of the SF-36 Health Survey scale. Our results are also in agreement with previous studies suggesting that the impairment in quality of life in obese patients increases together with obesity severity, but it affects mainly the physical consequences of the disease $(10,11)$, whereas the lack of correlation between BMI and psychological-social repercussion could be ascribed to a process of adaptation to the social environment (20).

Our study has some limitations. First, because only obese patients were enrolled, we cannot compare the construct validity of SF-36 Health Survey in obese patients with that in normal-weight and overweight subjects. Furthermore, our results have been obtained in a population of obese patients seeking medical treatment and cannot be inferred to the whole population of obese patients. Indeed, HRQL varies significantly across treatment modalities, with non-treatment-seeking patients having the best and patients seeking surgical treatment having the worst HRQL (28). However, patients seeking medical treatment in community programs have significantly impaired HRQL when compared with non-treatment seekers $(28-30)$. Thus, results from the 
present study suggest that health care professionals involved in the medical treatment of obese treatment-seeking outpatients should be aware of the possible implications of measuring HRQL with SF-36 Health Survey. Finally, we did not consider a measure of comorbidity in the present study, and we cannot rule out that overall comorbidity or select comorbid conditions may affect the construct validity of the SF-36 Health Survey or the relationship between BMI and main components. Future studies in this field should take into account the coexistence of disabling conditions, such as congestive heart failure and chronic obstructive pulmonary disease, which may significantly affect the relationship of BMI with either the derived factors or the traditional SF-36 subscales.

In conclusion, the main finding from this study is that in a very specific outpatient sample of obese treatment-seeking patients, the clustering of SF-36 Health Survey items in main components is not significantly different from the domain-based approach generally used, thus confirming the robustness of such a generic questionnaire in this specific condition, too. Despite that, the peculiar clustering of some of the SF-36 items within this sample and the relationship between increasing BMI and HRQL do have a distinctive feature, suggesting that the HRQL profile of subjects belonging to that population may be better described with alternative aggregation of the SF-36 Health Survey items or with disease-tailored questionnaires.

\section{Acknowledgments}

The QUOVADIS study is supported by an unrestricted grant from BRACCO Imaging Spa, Milan, Italy. A complete list of the QUOVADIS investigators has been previously published (31).

\section{References}

1. National Institutes of Health. The Practical Guide: Identification, Evaluation, and Treatment of Overweight and Obesity in Adults: Publication No. 00-4084. Bethesda, MD: NIH; 2000.

2. Kolotkin RL, Meter K, Williams GR. Quality of life and obesity. Obes Rev. 2001;2:219-29.

3. Ware JE Jr, Snow KK, Kosinski M, et al. SF-36 Health Survey: Manual and Interpretation Guide. Boston, MA: The Health Institute of New England Medical Center; 1993.

4. McHorney CA, Ware JE, Rogers W, et al. The validity and relative precision of MOS Short- and Long-Form Health Status Scales of Dartmouth COOP charts. Med Care. 1992; 30(Suppl 5):MS253-65.

5. Ware JE, Sherbourne CD. The MOS 36-Item Short-Form Health Survey (SF-36): I. Conceptual framework and item selection. Med Care. 1992;30:473-83.

6. Ware JE Jr, Kosinski M, Gandek B, et al. The factor structure of the SF-36 Health Survey in 10 countries: results from the IQOLA Project: International Quality of Life Assessment. J Clin Epidemiol. 1998;51:1159-65.
7. Wadden TA, Phelan S. Assessment of quality of life in obese individuals. Obes Res. 2002;10(Suppl 1):50-7S.

8. Fontaine KR, Cheskin LJ, Barofsky I. Health-related quality of life in obese persons seeking treatment. J Fam Pract. 1996;43:265-70.

9. Barofsky I, Fontaine KR, Cheskin LJ. Pain in the obese: impact on health-related-quality-of-life. Ann Beh Med. 1998; 19:408-10.

10. Yancy WS, Olsen MK, Westman EC, Bosworth HB, Edelman B. Relationship between obesity and health-related quality of life in men. Obes Res. 2002;10:1057-64.

11. Doll HA, Petersen SEK, Stewart-Brown SL. Obesity and physical and emotional well-being: associations between body mass index, chronic illness, and the physical and mental components of the SF-36 Questionnaire. Obes Res. 2000;8: $160-70$

12. Marchesini G, Bellini M, Natale S, et al. Psychiatric distress and health-related quality of life in obesity. Diabetes Nutr Metab. 2003;16:145-54.

13. Marchesini G, Solaroli E, Baraldi L, et al. Health-related quality of life in obesity: the role of eating behaviour. Diabetes Nutr Metab. 2000;13:156-64.

14. Ware JE, Kosinski M, Bayliss MS, et al. Comparison of methods for the scoring and statistical analysis of SF-36 health profile and summary measures: summary of results from the medical outcomes study. Med Care. 1995;33(Suppl 4):26479.

15. Apolone G, Mosconi P. The Italian SF-36 Health Survey: translation, validation and norming. J Clin Epidemiol. 1998; 51:1025-36.

16. Melchionda N, Marchesini G, Apolone G, Cuzzolaro M, Mannucci E, Grossi E, and the QUOVADIS Study Group. The QUOVADIS study: features of obese Italian patients seeking treatment at specialist centers. Diab Nutr Metab. 2003;16:115-24.

17. Gruijter DNM, Van der Kamp LJT. Statistical models in psychological and educational testing. Lisse, The Netherlands: Swets \& Zeitlinger; 1984.

18. Zwick W, Velicer W. Comparison of five rules for determining the number of components to retain. Psychol Bull. 1986; 99:432-42.

19. Patrick DL, Deyo RA. Generic and disease-specific measures in assessing health status and quality of life. Med Care. 1989;27(Suppl 3):S217-32.

20. Le Pen C, Levy E, Loos F, Banzet MN, Basdevant A. "Specific" scale compared with "generic" scale: a double measurement of the quality of life in a French community sample of obese subjects. J Epidemiol Community Health. 1998;52:445-50.

21. Blair SN, LaMonte MJ, Nichaman MZ. The evolution of physical activity recommendations: how much is enough? Am J Clin Nutr. 2004;79:913-20S

22. Hill JO, Wyatt HR. Role of physical activity in preventing and treating obesity. J Appl Physiol. 2005;99:765-70.

23. Jia H, Lubetkin EI. The impact of obesity on health-related quality-of-life in the general adult US population. $J$ Public Health (Oxf). 2005;27:156-64. 
24. Cilli M, De Rosa R, Pandolfi C, et al. Quantification of sub-clinical anxiety and depression in essentially obese patients and normal-weight healthy subjects. Eat Weight Disord. 2003;8:319-20.

25. Heo M, Allison DB, Faith MS, Zhu S, Fontaine KR. Obesity and quality of life: mediating effects of pain and comorbidities. Obes Res. 2003;11:209-16.

26. Marcus DA. Obesity and the impact of chronic pain. Clin J Pain. 2004;20:186-91.

27. Barofsky I, Fontaine KR, Cheskin LJ. Pain in the obese: impact on health-related quality-of-life. Ann Behav Med. 1997; 19:408-10.
28. Kolotkin RL, Crosby RD, Williams GR. Health-related quality of life varies among obese subgroups. Obes Res. 2002;10:748-56.

29. Foster GD, Kendall PC. The realistic treatment of obesity. Clin Psych Rev. 1994;14:701-36.

30. Fitzgibbon ML, Stolley MR, Kirschenbaum DS. Obese people who seek treatment have different characteristics than those who do not seek treatment. Health Psychol. 1993;12: 342-34.

31. Marchesini G, Melchionda N, Apolone G, et al. The metabolic syndrome in treatment-seeking obese persons. Metabolism. 2004;53:435-40. 